\title{
Final-state interactions in inclusive deep-inelastic scattering from the deuteron
}

\author{
W. Cosyn* \\ Department of Physics and Astronomy, Ghent University, Proeftuinstraat 86, B-9000 Gent, Belgium \\ and Department of Physics, Florida International University, Miami, Florida 33199, USA \\ W. Melnitchouk \\ Jefferson Lab, 12000 Jefferson Avenue, Newport News, Virginia 23606, USA \\ M. Sargsian \\ Department of Physics, Florida International University, Miami, Florida 33199, USA
}

(Received 16 November 2013; published 16 January 2014)

\begin{abstract}
We explore the role of final-state interactions (FSIs) in inclusive deep-inelastic scattering from the deuteron. Relating the inclusive cross section to the deuteron forward virtual Compton scattering amplitude, a general formula for the FSI contribution is derived in the generalized eikonal approximation, utilizing the diffractive nature of the effective hadron-nucleon interaction. The calculation uses a factorized model with a basis of three resonances with mass $W<2 \mathrm{GeV}$ and a continuum contribution for larger $W$ as the relevant set of effective hadron states entering the final-state interaction amplitude. The results show sizeable on-shell FSI contributions for Bjorken $x \gtrsim 0.6$ and $Q^{2} \lesssim 10 \mathrm{GeV}^{2}$, increasing in magnitude for lower $Q^{2}$, but vanishing in the high- $Q^{2}$ limit because of phase-space constraints. The off-shell rescattering contributes at $x \gtrsim 0.8$ and is taken as an uncertainty on the on-shell result.
\end{abstract}

DOI: 10.1103/PhysRevC.89.014612

PACS number(s): 11.80.Fv, 13.60.Hb, 24.85.+p, 25.30.-c

\section{INTRODUCTION}

Inclusive deep-inelastic scattering (DIS) from the deuteron has for a considerable time been the main source of information on the partonic structure of the neutron [1-5]. Recently, there has been growing emphasis placed on such extractions at large values of the momentum fraction $x$ carried by the partons [6-11]. When combined with the more readily available proton data, and assuming charge symmetry of the nucleon's parton distribution functions (PDFs), one can directly reconstruct the individual $u$ and $d$ quark PDFs that dominate nucleon structure at large values of $x$.

At high $x \quad(x \gtrsim 0.5)$ the difference between the parton structure of the proton and neutron grows and becomes rather sensitive to the underlying QCD dynamics generating high-momentum partons in the nucleon. Unfortunately, in this region the extraction of parton distributions in the neutron from inclusive deuteron DIS data becomes increasingly complicated by the effects of nuclear corrections. Within the nuclear impulse approximation, in which the scattering takes place incoherently from individual nucleons bound in the nucleus, these effects include nuclear Fermi motion and binding, relativistic and off-shell corrections, and non-nucleonic components of the deuteron wave function. Considerable effort has been made over the years to understand these effects quantitatively (see, e.g., Refs. [12-20]).

Beyond the impulse approximation, rescattering effects can also play an important role in the DIS process; for example, multiple scattering of the beam from two (or more, for larger nuclei) nucleons can give rise to nuclear shadowing corrections

\footnotetext{
*Wim.Cosyn@UGent.be
}

at small $x$ [21-26]. The interaction of the hadronic debris of the struck nucleon with the spectator nucleon in the final state, however, can give contributions also at higher $x$ values. These final-state interaction (FSI) effects are generally considered to be small in inclusive DIS at moderate to small values of $x$, where, for large invariant mass $W$ produced in a high-energy collision, the quantum phase space in the final state of the reaction is unrestricted. As a result, the closure relation can be applied to the sum over the final states, enabling these to be represented through quark degrees of freedom or, in other words, quark-hadron duality is expected to hold in these kinematics [27]. Dynamically, this is consistent with the picture in which the struck quark hadronizes well after leaving the target, so that the final-state rearrangement does not influence the initial-state probability distribution of the interacting partons [28].

The situation can be quite different for large- $x$ and finite- $W$ kinematics, in which the condition for the closure approximation (or duality) is not fully satisfied. However, even if finite FSI effects are expected here, estimating their contribution requires knowledge about the composition and internal distribution of momentum of the final hadronic state in the DIS process, a problem which remains very challenging. The structure of the DIS final state can be examined by considering the production of specific hadrons in coincidence with the scattered electron [29-33]. In particular, a promising avenue has been the study of the distribution of slow tagged protons (with momentum up to $\sim 500 \mathrm{MeV}$ ) in semi-inclusive DIS (SIDIS) from the deuteron [34,35].

Recently, an approach was developed [32,33] for calculating the SIDIS reaction from the deuteron that accounted for FSI effects based on general properties of high-energy diffractive scattering. The underlying assumption was that, 
because of restricted phase space (finite values of $W$ and $Q^{2}$ ), the minimal Fock-state component of the wave function can be used to describe DIS from the bound nucleon. In this case the scattered state consists of three outgoing valence quarks whose rescattering from the spectator nucleon is parametrized in the form of a $Q^{2}$ - and $W$-dependent diffractive amplitude. The results of this approach showed good agreement with the most recent SIDIS data from Hall B at Jefferson Lab [34], especially in the description of the rise of the FSI effects in the forward direction of the spectator nucleon production. Comparison with the data also allowed one to extract the parametric dependence of the diffractive rescattering amplitudes, which increase with $W$ and decrease with $Q^{2}$.

A related calculation was performed in Refs. [29,31], also using an eikonal approximation to estimate the FSI amplitude. Here a Glauber model was employed with a time-dependent debris-nucleon cross section, including, in addition, the contribution from the target fragmentation region to the SIDIS cross section, and calculations for SIDIS off ${ }^{12} \mathrm{C}$ were also presented. Good agreement with the deuteron SIDIS data [34] was found in a wide region of backward nucleon emission, whereas, as expected, a traditional Glauber approach was difficult to accommodate at forward spectator nucleon kinematics. The kinematical region of slow spectator protons in the backward hemisphere was found to have small FSI contributions, making it useful for neutron structure function extraction. Fast protons in perpendicular kinematics, however, yielded large FSI effects, making this region suited for the study of hadronization mechanisms. For fast spectator protons the contribution from the target fragmentation region was found to become significant, especially in the forward hemisphere.

Building on the knowledge gained from the semi-inclusive analyses, in this paper we extend the approach of Refs. [32,33] to inclusive DIS from the deuteron, over a similar range of $Q^{2}$ and $W$ that was covered in the SIDIS kinematics. The observation from the SIDIS studies [32,33] that the FSI structure is consistent with diffractive scattering allows the generalized eikonal approximation (GEA) model to be extended [32,36,37] to the inclusive DIS reaction through the optical theorem, relating the inclusive cross section to the imaginary part of the forward $\gamma^{*} D$ Compton scattering amplitude. The general correspondence between the inclusive DIS cross section and the forward Compton is derived in Sec. II. The Compton scattering amplitude is then computed, first in the plane-wave Born approximation and then in the presence of final-state hadronic interactions, taking into account both on-shell and off-shell contributions in the rescattering amplitude. The results presented in Sec. II are rather general, relying only on the diffractive nature of the FSI. The specific model used to obtain the numerical estimates of FSI effects is introduced in Sec. III, where its main assumptions and approximations are highlighted. These include a factorized approach for the hadronic currents in the FSI amplitude and a three-resonance model combined with a DIS continuum region distribution at large $W$ for the states that contribute to the FSI. The numerical results for the FSI effects are presented in Sec. IV, and conclusions are drawn in Sec. V.

\section{THEORETICAL FRAMEWORK}

In this section we present the definitions for cross sections and the nuclear hadronic tensor corresponding to the inclusive scattering of an electron $e$ from a nucleus $A$,

$$
e\left(k_{i}\right)+A\left(p_{A}\right) \rightarrow e^{\prime}\left(k_{f}\right)+X\left(p_{X}\right),
$$

where $k_{i}$ and $k_{f}$ are the four-momenta of the initial- and final-state electrons, and $p_{A}$ and $p_{X}$ are the four-momenta of the target nucleus and the produced hadronic system $X$, respectively. While the formal results derived here will be valid for any nucleus $A$, in the actual calculations we specialize to the case of the deuteron.

We base our derivations on the relationship between the inclusive electroproduction cross section and the imaginary part of the amplitude of forward virtual Compton scattering off the nucleus. The advantage of such an approach is that the amplitudes accounting for the FSI effects will self-consistently satisfy the unitarity conditions for inelastic rescattering. An alternative approach would be to introduce FSI effects in the $\gamma^{*} D \rightarrow X$ scattering amplitude and apply Abramovsky, Gribov, Kancheli (AGK)-type cutting rules [38] in the calculation of the cross section to restore unitarity. In our approach we explicitly identify the Born and FSI terms of the inclusive electroproduction cross section with the impulse approximation and FSI contribution in the forward Compton scattering amplitude, with the latter calculated in the GEA.

\section{A. Inclusive cross section and forward nuclear virtual Compton scattering}

Neglecting electron masses, we define the differential DIS cross section as

$$
\begin{aligned}
d \sigma= & \frac{1}{\left.4 \sqrt{\left(k_{i} p_{A}\right.}\right)^{2}} \sum_{X} \overline{\sum_{i, f}}|\mathcal{M}|^{2}(2 \pi)^{4} \delta^{(4)}\left(q+p_{A}-p_{X}\right) \\
& \times \frac{d^{3} \boldsymbol{k}_{f}}{(2 \pi)^{3} 2 \epsilon_{f}} \frac{d^{3} \boldsymbol{p}_{X}}{(2 \pi)^{3} 2 E_{X}},
\end{aligned}
$$

where $\epsilon_{f}$ and $E_{X}$ are the energies of the final electron and hadronic state $X, q=p_{X}-p_{A}=k_{i}-k_{f}$ is the fourmomentum transfer to the target, and we average the square of the scattering amplitude $\mathcal{M}$ over the initial spins of the electron and nucleus and sum over the scattered electron spins. The formal sum $\sum_{X}$ includes all possible final states $|X\rangle$ and integrates over the distributions of their internal momenta. Using the phase-space identity for $p_{X}$,

$$
\frac{d^{3} \boldsymbol{p}_{X}}{2 E_{X}}=d^{4} p_{X} \delta\left(p_{X}^{2}-W_{X}^{2}\right) \theta\left(E_{X}\right),
$$

we can eliminate the four-dimensional $\delta$ function with $d^{4} p_{X}$ and express the differential cross section as

$$
\begin{aligned}
\frac{d \sigma}{d \epsilon_{f} d \Omega_{f}}= & \frac{1}{(4 \pi)^{2}} \frac{1}{2 M_{A}} \frac{\epsilon_{f}}{\epsilon_{i}} \\
& \times \sum_{X} \overline{\sum_{i, f}}|\mathcal{M}|^{2} \delta\left[\left(p_{A}+q\right)^{2}-W_{X}^{2}\right] \theta\left(E_{X}\right) .
\end{aligned}
$$


Here $W_{X}^{2} \equiv p_{X}^{2}$ is the invariant mass of the produced hadronic state $X$ and $\epsilon_{i}$ is the incoming electron energy. Introducing the DIS interaction vertex $\Gamma_{A X}^{\mu}$ between initial nuclear ground sate $\left|\Psi_{A}\right\rangle$ and final state $\left|\Phi_{X}\right\rangle$, one can represent the matrix element $\mathcal{M}$ of the scattering as the product of leptonic $\left(J_{\mu}^{e}\right)$ and nuclear $\left(J_{A X}^{\mu}\right)$ currents,

$$
-i \mathcal{M}=-\frac{i e^{2}}{q^{2}} J_{\mu}^{e}\left(k_{f}, s_{e^{\prime}} ; k_{i}, s_{e}\right) J_{A X}^{\mu}\left(p_{A}, s_{A} ; p_{X}, s_{X}\right),
$$

where $J_{\mu}^{e}=\bar{u}\left(k_{f}, s_{e^{\prime}}\right) \gamma_{\mu} u\left(k_{i}, s_{e}\right)$ and $J_{A X}^{\mu}\left(p_{A}, s_{A} ; p_{X}, s_{X}\right)=$ $\left\langle\Phi\left(p_{X}, s_{X}\right)\left|\Gamma_{A X}^{\mu}\right| \Psi_{A}\left(p_{A}, s_{A}\right)\right\rangle$. Here $s_{e}\left(s_{e^{\prime}}\right), s_{X}$, and $s_{A}$ are the spins of the incoming (final) electron, hadronic state $X$, and nucleus $A$, respectively. In terms of the currents, the differential cross section in Eq. (4) can be written in terms of leptonic $\left(L_{\mu \nu}\right)$ and hadronic $\left(W_{A}^{\mu \nu}\right)$ tensors,

$$
\frac{d \sigma}{d \epsilon_{f} \Omega_{f}}=\frac{\alpha^{2}}{Q^{4}} \frac{\epsilon_{f}}{\epsilon_{i}} L_{\mu \nu} W_{A}^{\mu \nu},
$$

where $\alpha$ is the electromagnetic coupling. The leptonic tensor is given by

$$
L_{\mu \nu}=\frac{1}{2} \sum_{s_{e}, s_{e^{\prime}}} J_{\mu}^{e \dagger} J_{\nu}^{e}=2\left(k_{i \mu} k_{f \nu}+k_{f \mu} k_{i \nu}+\frac{q^{2}}{2} g_{\mu \nu}\right),
$$

while the hadronic tensor can be formally written as

$$
\begin{aligned}
W_{A}^{\mu \nu}= & \frac{1}{2 M_{A}} \frac{1}{\left(2 j_{A}+1\right)} \\
& \times \sum_{X} \sum_{s_{A}, s_{X}} J_{A X}^{\mu \dagger}\left(p_{A}, s_{A} ; p_{X}, s_{X}\right) J_{A X}^{v}\left(p_{A}, s_{A} ; p_{X}, s_{X}\right) \\
& \times \delta\left[\left(p_{A}+q\right)^{2}-W_{X}^{2}\right] \theta\left(E_{X}\right)
\end{aligned}
$$

where $j_{A}$ is the total spin of the nucleus $A$.

Expressing the DIS differential cross section through the nuclear hadronic tensor makes the application of the optical theorem rather straightforward. From the nuclear virtual Compton scattering amplitude shown in Fig. 1 one observes that the imaginary part of the intermediate-state propagator, with the condition $E_{X}>0$, corresponds to $\pi \delta\left[\left(p_{A}+q\right)^{2}-W_{X}^{2}\right]$. This gives for the imaginary part of the Compton amplitude in the forward

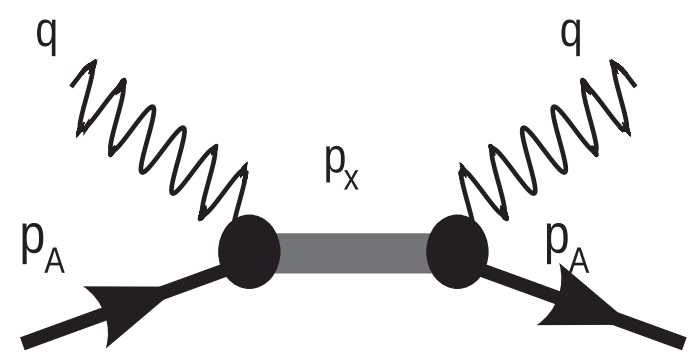

FIG. 1. Forward virtual Compton scattering amplitude from a nucleus $A$, with $q$ and $p_{A}$ the photon and target four-momenta and $p_{X}$ the four-momentum of the produced state $X$. direction $(t=0)$,

$$
\begin{aligned}
\operatorname{Im} & \mathcal{A}_{\gamma^{*} A}^{\mu \nu}(t=0) \\
= & \sum_{X} J_{A X}^{\mu \dagger}\left(p_{A}, s_{A} ; p_{X}, s_{X}\right) J_{A X}^{v}\left(p_{A}, s_{A} ; p_{X}, s_{X}\right) \\
& \quad \times \pi \delta\left[\left(p_{A}+q\right)^{2}-W_{X}^{2}\right] \theta\left(E_{X}\right),
\end{aligned}
$$

where $s_{A}=s_{A^{\prime}}$ from the forward elastic scattering condition. Comparing Eqs. (8) and (9) one obtains the optical theorem relation between the nuclear hadronic tensor and the forward nuclear Compton scattering amplitude,

$$
W_{A}^{\mu \nu}=\frac{1}{2 \pi M_{A}} \frac{1}{\left(2 j_{A}+1\right)} \sum_{s_{A}} \operatorname{Im} \mathcal{A}_{\gamma^{*} A}^{\mu \nu}(t=0) .
$$

Although the above discussion holds for an arbitrary nucleus $A$, we shall now focus on the specific case of the deuteron. At large $Q^{2}$ and for deuteron internal momenta up to $700 \mathrm{MeV}$ (see, e.g., Ref. [39]) one expects the virtual photon scattering from the deuteron target to take place from an individual nucleon bound in the nucleus. This allows us to express the Compton scatting amplitude as a sum of two terms, as illustrated in Fig. 2. The first (Born) term represents the propagation of the state $X^{\prime}$ resulting from the $\gamma^{*}$-bound nucleon scattering, without interacting with the spectator nucleon [Fig. 2(a)]. The second (rescattering) term corresponds to the produced hadronic state $\left(X_{1}\right)$ interacting with the spectator nucleon $\left(S_{1}\right)$ in the intermediate state of the Compton scattering [Fig. 2(b)]. The latter diagram is responsible for the FSI contribution to inclusive DIS.

\section{B. Born term}

For the Born diagram of the forward Compton scattering amplitude from the deuteron, following the prescription of the effective Feynman diagram rules for inelastic scattering [32],
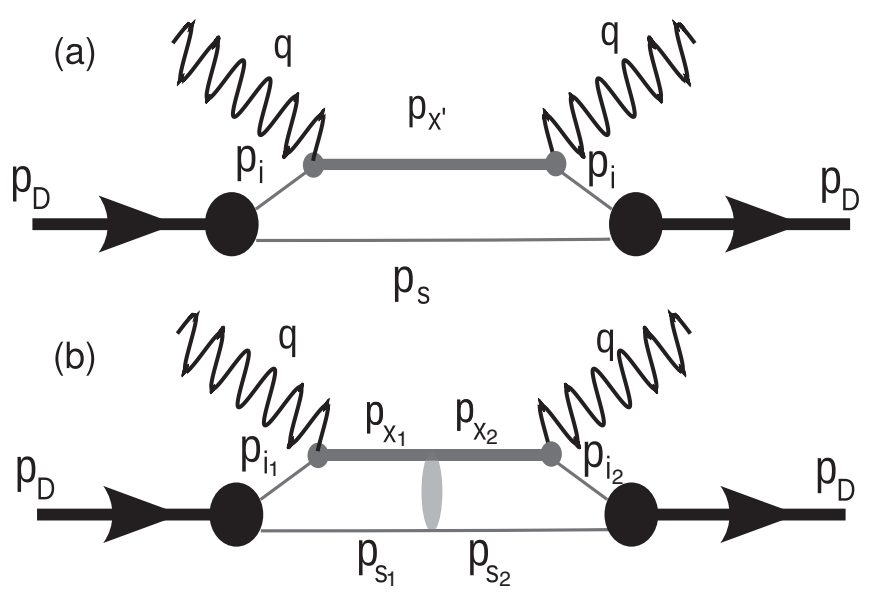

FIG. 2. Forward virtual Compton scattering amplitude for the deuteron, composed of (a) the Born diagram and (b) the rescattering contribution. The gray blob in the intermediate state represents the effective rescattering interaction of the hadronic debris $\left(X_{1}\right)$ and the spectator nucleon $\left(S_{1}\right)$ to the final hadronic state $\left(X_{2}\right)$ and nucleon $\left(S_{2}\right)$. The deuteron momentum in the Born diagram is given by $p_{D}=$ $p_{i}+p_{s}$ and in the FSI diagram by $p_{D}=p_{i_{1}}+p_{s_{1}}=p_{i_{2}}+p_{s_{2}}$. 
the plane-wave (pw) amplitude can be written as

$$
\begin{aligned}
\mathcal{A}_{\mathrm{pw}}^{\mu \nu}= & \sum_{N, X^{\prime}} \int \frac{d^{4} p_{s}}{i(2 \pi)^{4}}\left(\chi^{s_{D}}\right)^{\dagger} \Gamma_{D N N}^{\dagger} \frac{\not p_{i}+m}{p_{i}^{2}-m^{2}+i \epsilon} \Gamma_{\gamma N X^{\prime}}^{\mu \dagger} \\
& \times \frac{G\left(p_{X^{\prime}}\right)}{p_{X^{\prime}}^{2}-m_{X^{\prime}}^{2}+i \epsilon} \\
& \times \frac{\not p_{s}+m}{p_{s}^{2}-m^{2}+i \epsilon} \Gamma_{\gamma N X^{\prime}}^{v} \frac{\not p_{i}+m}{p_{i}^{2}-m^{2}+i \epsilon} \Gamma_{D N N} \chi^{s_{D}},
\end{aligned}
$$

where $p_{i}$ is the four-momentum of the initial, off-shell nucleon and $p_{s}$ is the four-momentum of the spectator nucleon, both with mass $m$. The sum runs over all possible intermediate states $X^{\prime}$ and the proton and neutron contribution. The inelastic intermediate state is characterized by the momentum $p_{X^{\prime}}=$ $p_{D}+q-p_{s}$ and mass $m_{X^{\prime}}$. The function $G\left(p_{X^{\prime}}\right)$ describes the Green's function of the intermediate state $X^{\prime}, \Gamma_{\gamma N X^{\prime}}^{\mu}$ is the photon-nucleon vertex, and $\Gamma_{D N N}$ denotes the $D N N$ vertex function, with $\chi^{s_{D}}$ the deuteron spin-wave function for spin projection $s_{D}$. In the virtual nucleon approximation (VNA) $[32,40]$, the loop integration over $d p_{s}^{0}$ is performed by retaining only the positive energy, on-mass-shell contribution of the spectator nucleon propagator,

$$
\int \frac{d p_{s}^{0}}{p_{s}^{2}-m^{2}+i \epsilon} \longrightarrow-i \frac{\pi}{E_{s}}
$$

where $E_{s}=\sqrt{m^{2}+p_{s}^{2}}$ is the spectator nucleon energy. Conservation of energy requires that the energy of the interacting, off-shell nucleon is then equal to $E_{i}=M_{D}-E_{s}$.

It is convenient in the VNA to introduce the deuteron wave function $\Psi_{D}^{s_{D}}\left(p_{1}, s_{1} ; p_{2}, s_{2}\right)$ for the case of one nucleon $\left(p_{1}\right)$ being off shell and one nucleon $\left(p_{2}\right)$ on shell [32,40-43],

$$
\Psi_{D}^{s_{D}}\left(p_{1}, s_{1} ; p_{2}, s_{2}\right)=-\frac{\bar{u}\left(p_{1}, s_{1}\right) \bar{u}\left(p_{2}, s_{2}\right) \Gamma_{D N N} \chi^{s_{D}}}{\left(p_{1}^{2}-m^{2}\right) \sqrt{2} \sqrt{(2 \pi)^{3} 2 E_{2}}}
$$

where $E_{2}=\sqrt{m^{2}+p_{2}^{2}}$, and we take the masses of the two nucleons to be equal, $m_{1}=m_{2}=m$. This then allows the pw amplitude to be expressed as

$$
\begin{aligned}
\mathcal{A}_{\mathrm{pw}}^{\mu \nu}= & -2 \sum_{N, X^{\prime}} \sum_{s_{i}, s_{i}^{\prime}, s_{s}} \int d^{3} \boldsymbol{p}_{s} \Psi_{D}^{s_{D} \dagger}\left(p_{i}, s_{i}^{\prime} ; p_{s}, s_{s}\right) \bar{u}\left(p_{i}, s_{i}^{\prime}\right) \Gamma_{\gamma N X^{\prime}}^{\mu \dagger} \\
& \times \frac{G\left(p_{X^{\prime}}\right)}{p_{X^{\prime}}^{2}-m_{X^{\prime}}^{2}+i \epsilon} \Gamma_{\gamma N X^{\prime}}^{\nu} u\left(p_{i}, s_{i}\right) \Psi_{D}^{s_{D}}\left(p_{i}, s_{i} ; p_{s}, s_{S}\right),
\end{aligned}
$$

where $s_{i}, s_{i}^{\prime}$ are the spins of the off-shell nucleons and $s_{s}$ is the spin of the spectator on-shell nucleon. In general, the propagator of the inelastic intermediate state $X^{\prime}$ can be represented as a sum of on-shell and off-shell contributions
[32],

$$
\begin{aligned}
& \frac{G\left(p_{X^{\prime}}\right)}{p_{X^{\prime}}^{2}-m_{X^{\prime}}^{2}+i \epsilon} \\
& \approx \frac{\sum_{s_{X^{\prime}}}\left|p_{X^{\prime}}, s_{X^{\prime}}\right\rangle\left\langle p_{X^{\prime}}, s_{X^{\prime}}\right|}{p_{X^{\prime}}^{2}-m_{X^{\prime}}^{2}+i \epsilon} \\
& =-i \pi \sum_{s_{X^{\prime}}}\left|p_{X^{\prime}}, s_{X^{\prime}}\right\rangle\left\langle p_{X^{\prime}}, s_{X^{\prime}}\right| \delta\left(p_{X^{\prime}}^{2}-m_{X^{\prime}}^{2}\right) \theta\left(E_{X^{\prime}}\right) \\
& \quad+\mathcal{P} \frac{\sum_{s_{X^{\prime}}}\left|p_{X^{\prime}}, s_{X^{\prime}}\right\rangle\left\langle p_{X^{\prime}}, s_{X^{\prime}}\right|}{p_{X^{\prime}}^{2}-m_{X^{\prime}}^{2}}
\end{aligned}
$$

where in the off-shell term the symbol $\mathcal{P}$ denotes the Cauchy principal value integration. Because the imaginary part of the Compton scattering amplitude is defined by the on-shell part of the inelastic state $X^{\prime}$ propagator, substituting Eq. (15) into Eq. (14) and averaging over the deuteron polarizations, one obtains

$$
\begin{aligned}
& \frac{1}{3} \sum_{s_{D}} \operatorname{Im} \mathcal{A}_{\mathrm{pw}}^{\mu \nu} \\
& =\pi \int d^{3} p_{s} \sum_{N, X^{\prime}} \sum_{s_{i}, s_{X^{\prime}}} J_{\gamma N X^{\prime}}^{\mu \dagger}\left(p_{i}, s_{i} ; p_{X^{\prime}}, s_{X^{\prime}}\right) \\
& \quad \times J_{\gamma N X^{\prime}}^{v}\left(p_{i}, s_{i} ; p_{X^{\prime}}, s_{X^{\prime}}\right) \delta\left(p_{X^{\prime}}^{2}-m_{X^{\prime}}^{2}\right) \theta\left(E_{X^{\prime}}\right) S\left(p_{s}\right)
\end{aligned}
$$

where the deuteron momentum distribution is defined as

$$
S\left(p_{s}\right)=\frac{1}{3} \sum_{s_{D}, s_{s}, s_{i}}\left|\Psi_{D}^{s_{D}}\left(p_{i}, s_{i} ; p_{s}, s_{s}\right)\right|^{2} .
$$

Introducing the nucleon hadronic tensor in analogy with Eq. (8),

$$
\begin{aligned}
W_{N}^{\mu \nu}= & \frac{1}{2 m} \frac{1}{2} \sum_{X^{\prime}} \sum_{s_{i}, s_{X^{\prime}}} J_{\gamma N X^{\prime}}^{\mu \dagger}\left(p_{i}, s_{i} ; p_{X^{\prime}}, s_{X^{\prime}}\right) \\
& \times J_{\gamma N X^{\prime}}^{v}\left(p_{i}, s_{i} ; p_{X^{\prime}}, s_{X^{\prime}}\right) \delta\left(p_{X^{\prime}}^{2}-m_{X^{\prime}}^{2}\right) \theta\left(E_{X^{\prime}}\right),
\end{aligned}
$$

and using it in Eq. (16), from the optical theorem relation in Eq. (10) one obtains for the deuteron hadronic tensor,

$$
W_{D}^{\mu \nu}=\frac{2 m}{M_{D}} \sum_{N} \int d^{3} \boldsymbol{p}_{s} W_{N}^{\mu \nu} S\left(p_{s}\right)
$$

where the sum is over the nucleons $N=p, n$. This corresponds to the usual convolution model of inclusive DIS from the deuteron $[13,17,18,44]$.

\section{Final-state interaction contribution}

Having outlined the basic derivation of the Born contribution to DIS from the deuteron, we are now able to proceed with the calculation of the FSI corrections to the inclusive deuteron structure functions. The strategy is to use the GEA to compute the rescattering contribution in Fig. 2(b) to the forward Compton scattering amplitude and relate it to the inclusive DIS process via the optical theorem [Eq. (10)]. Note that in the eikonal approximation the hadronic rescattering vertex is an effective vertex related to the hadronic scattering amplitude. Consequently, only the FSI amplitude in Fig. 2(b) 


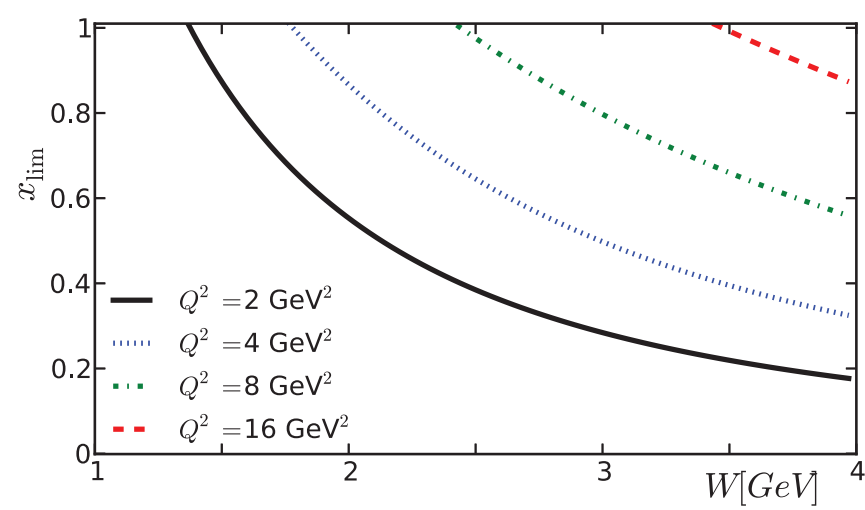

FIG. 3. (Color online) Maximum value of Bjorken $x$ allowed for the application of the eikonal approximation as a function of the invariant mass $W$ of the state $X^{\prime}$.

needs to be considered, where a sum over all intermediate states $X_{1}$ and $X_{2}$ is taken, and higher order rescattering contributions are included in the effective rescattering vertex. Two conditions, however, must be satisfied for the GEA to be valid in the calculation of the FSI contribution.

(1) The intermediate state can be characterized as an effective hadronic state whose interaction with the spectator nucleon can have attributes of the $h N$ interaction. Such states comprise any intermediate-state resonances that can be generated at the first $\gamma^{*} N$ vertex in Fig. 2(b).
(2) The produced state $X_{1}$ is energetic enough for the eikonal approximation to be valid for $X_{1} N$ rescattering, and it can be described by a diffractive amplitude.

For the second condition we can use the empirical observation that in hadron-nucleon scattering the eikonal approximation holds for hadron momenta $\gtrsim 500 \mathrm{MeV}$ [45-47] in a frame where the nucleon is at rest. This can be used to define the quantity $x_{\lim }$ as the maximum value of the Bjorken scaling variable $x=Q^{2} / 2 m q_{0}$ at a given $Q^{2}$ for which the state $X^{\prime}$ in the rescattering system has a momentum of at least $500 \mathrm{MeV}$. This value of $x_{\text {lim }}$ can be found from the relation

$$
s_{X^{\prime} N}=\left(p_{D}+q\right)^{2}=m^{2}+m_{X^{\prime}}^{2}+2 m E_{X^{\prime}}
$$

where $E_{X^{\prime}}$ is the energy of the state $X^{\prime}$ in a frame where the "spectator" nucleon $\left[S_{1}\right.$ in Fig. 2(b)] is at rest. In Fig. 3 we show $x_{\text {lim }}$ as a function of the invariant mass $W$ of $X^{\prime}$ taking part in the rescattering for several values of $Q^{2}$. For values of $Q^{2}$ up to $5 \mathrm{GeV}^{2}$ care should be taken when $x>0.5$ for values of $W$ higher than $2 \mathrm{GeV}$ entering in the rescattering amplitude.

Assuming now that the chosen kinematics is appropriate for the application of the eikonal approximation and that the intermediate states $X$ are identified, one can apply the effective Feynman diagram rules for the rescattering diagram of Fig. 2(b) to obtain the FSI contribution to the virtual Compton amplitude,

$$
\begin{aligned}
\mathcal{A}_{\mathrm{FSI}}^{\mu \nu}= & \sum_{N, X_{1}, X_{2}} \int \frac{d^{4} p_{s_{1}}}{i(2 \pi)^{4}} \frac{d^{4} p_{s_{2}}}{i(2 \pi)^{4}}\left(\chi^{s_{D}}\right)^{\dagger} \Gamma_{D N N}^{\dagger} \frac{\not p_{i_{2}}+m}{p_{i_{2}}^{2}-m^{2}+i \epsilon} \Gamma_{\gamma N X_{2}}^{\mu \dagger} \frac{G\left(p_{X_{2}}\right)}{\left(p_{X_{2}}^{2}-m_{X_{2}}^{2}+i \epsilon\right)} \\
& \times \frac{\not p_{s_{2}}+m}{p_{s_{2}}^{2}-m^{2}+i \epsilon} F_{N X_{1}, N X_{2}} \frac{G\left(p_{X_{1}}\right)}{\left(p_{X_{1}}^{2}-m_{X_{1}}^{2}+i \epsilon\right)} \frac{\not p_{s_{1}}+m}{p_{s_{1}}^{2}-m^{2}+i \epsilon} \Gamma_{\gamma N X_{1}}^{\nu} \frac{\not p_{i_{1}}+m}{p_{i_{1}}^{2}-m^{2}+i \epsilon} \Gamma_{D N N} \chi^{s_{D}},
\end{aligned}
$$

where $F_{N X_{1}, N X_{2}}$ is the $N X_{1} \rightarrow N X_{2}$ effective scattering amplitude. To evaluate the FSI amplitude, we first integrate over $p_{s_{1}}^{0}$ and $p_{s_{2}}^{0}$ through the positive energy poles, as in Eq. (12). According to the VNA, we can then use the on-mass-shell decomposition of the virtual nucleon propagators, with their off-shell four-momenta $p_{i_{1}}$ and $p_{i_{2}}$ defined through four-momentum conservation at the $D N N$ vertices, to introduce the deuteron wave functions of Eq. (13) into the amplitude in Eq. (21). Finally, because of the large momenta involved in the propagators of the intermediate states $X_{j}(j=1,2)$, an on-shell relation for the Green's function $G\left(p_{X_{j}}\right)=\sum_{s_{X_{j}}}\left|p_{X_{j}}, s_{X_{j}}\right\rangle\left\langle p_{X_{j}}, s_{X_{j}}\right|$ can be used, which allows the FSI amplitude to be written as

$$
\begin{aligned}
\mathcal{A}_{\mathrm{FSI}}^{\mu \nu}= & 2(2 \pi)^{3} \sum_{N, X_{1}, X_{2}} \sum_{\substack{s_{i_{1}}, s_{s_{1}}, s_{X_{1}} \\
s_{i_{2}}, s_{s_{2}}, s_{X_{2}}}} \int \frac{d^{3} \boldsymbol{p}_{s_{1}}}{(2 \pi)^{3}} \frac{d^{3} \boldsymbol{p}_{s_{2}}}{(2 \pi)^{3}} \frac{\Psi_{D}^{s_{D} \dagger}\left(p_{i_{2}}, s_{i_{2}} ; p_{s_{2}}, s_{s_{2}}\right)}{\sqrt{2 E_{s_{2}}}} \frac{J_{\gamma N X_{2}}^{\mu \dagger}\left(p_{i_{2}}, s_{i_{2}} ; p_{X_{2}}, s_{X_{2}}\right)}{p_{X_{2}}^{2}-m_{X_{2}}^{2}+i \epsilon} \\
& \times\left\langle p_{X_{2}}, s_{X_{2}} ; p_{s_{2}}, s_{s_{2}}\left|F_{N X_{1}, N X_{2}}\right| p_{X_{1}}, s_{X_{1}} ; p_{s_{1}}, s_{S_{1}}\right\rangle \\
J_{\gamma N X_{1}}^{\nu}\left(p_{i_{1}}, s_{i_{1}} ; p_{X_{1}}, s_{X_{1}}\right) & \frac{\Psi_{D}^{s_{D}}\left(p_{i_{1}}, s_{i_{1}} ; p_{s_{1}}, s_{S_{1}}\right.}{p_{X_{1}}-m_{X_{1}}^{2}+i \epsilon} .
\end{aligned}
$$

In the following it is useful to write the product of the denominators of the intermediate inelastic-state propagators as

$$
\begin{aligned}
\frac{1}{\left(p_{X_{2}}^{2}-m_{X_{2}}^{2}+i \epsilon\right)} \frac{1}{\left(p_{X_{1}}^{2}-m_{X_{1}}^{2}+i \epsilon\right)} & =\left[-i \pi \delta\left(p_{X_{2}}^{2}-m_{X_{2}}^{2}\right)+\frac{\mathcal{P}}{p_{X_{2}}^{2}-m_{X_{2}}^{2}}\right]\left[-i \pi \delta\left(p_{X_{1}}^{2}-m_{X_{1}}^{2}\right)+\frac{\mathcal{P}}{p_{X_{1}}^{2}-m_{X_{1}}^{2}}\right] \\
& =-\pi^{2} \delta\left(p_{X_{1}}^{2}-m_{X_{1}}^{2}\right) \delta\left(p_{X_{2}}^{2}-m_{X_{2}}^{2}\right)+\frac{\mathcal{P}}{p_{X_{1}}^{2}-m_{X_{1}}^{2}} \frac{\mathcal{P}}{p_{X_{2}}^{2}-m_{X_{2}}^{2}},
\end{aligned}
$$


where the imaginary cross terms cancel exactly because of energy-momentum conservation at the rescattering vertices in the diagram of Fig. 2(b). This decomposition allows the amplitude in Eq. (22) to be separated into two terms containing on-shell and off-shell contributions to the rescattering amplitude $F_{N X_{1}, N X_{2}}$. It is also worth mentioning that the first part contains half-off-shell inelastic electromagnetic currents, while in the second part these are fully off shell. The first part of the decomposition (23) yields the "on-shell" component of the FSI contribution to the deuteron hadronic tensor,

$$
\begin{aligned}
W_{\mathrm{FSI}}^{\mu \nu(\mathrm{on})}= & -\frac{\pi(2 \pi)^{3}}{3 M_{D}} \sum_{N, X_{1}, X_{2}} \sum_{\text {spins }} \operatorname{Im} \int \frac{d^{3} \boldsymbol{p}_{s_{1}}}{(2 \pi)^{3}} \frac{d^{3} \boldsymbol{p}_{s_{2}}}{(2 \pi)^{3}} \frac{\Psi_{D}^{s_{D} \dagger}\left(p_{i_{2}}, s_{i_{2}} ; p_{s_{2}}, s_{S_{2}}\right) \Psi_{D}^{s_{D}}\left(p_{i_{1}}, s_{i_{1}} ; p_{s_{1}}, s_{S_{1}}\right)}{2 \sqrt{E_{S_{2}} E_{S_{1}}}} \\
& \times\left\langle p_{X_{2}}, s_{X_{2}} ; p_{s_{2}}, s_{S_{2}}\left|F_{N X_{1}, N X_{2}}^{(\mathrm{on})}\right| p_{X_{1}}, s_{X_{1}} ; p_{s_{1}}, s_{S_{1}}\right\rangle J_{\gamma N X_{2}}^{\mu \dagger}\left(p_{i_{2}}, s_{i_{2}} ; p_{X_{2}}, s_{X_{2}}\right) J_{\gamma N X_{1}}^{v}\left(p_{i_{1}}, s_{i_{1}} ; p_{X_{1}}, s_{X_{1}}\right) \\
& \times \delta\left(p_{X_{1}}^{2}-m_{X_{1}}^{2}\right) \delta\left(p_{X_{2}}^{2}-m_{X_{2}}^{2}\right),
\end{aligned}
$$

where the spin summation includes the sum over $s_{i_{1}}, s_{s_{1}}, s_{X_{1}}, s_{i_{2}}, s_{s_{2}}, s_{X_{2}}$, and $s_{D}$. Finally, the second term in Eq. (23) enters with opposite sign and represents the contribution of the off-shell part of the FSI contribution to the deuteron hadronic tensor,

$$
\begin{aligned}
W_{\mathrm{FSI}}^{\mu \nu \text { (off) }}= & \frac{(2 \pi)^{3}}{3 \pi M_{D}} \sum_{N, X_{1}, X_{2}} \sum_{\text {spins }} \operatorname{Im} \int_{\mathcal{P}} \frac{d^{3} \boldsymbol{p}_{s_{1}}}{(2 \pi)^{3}} \frac{d^{3} \boldsymbol{p}_{s_{2}}}{(2 \pi)^{3}} \frac{\Psi_{D}^{s_{D} \dagger}\left(p_{i_{2}}, s_{i_{2}} ; p_{s_{2}}, s_{S_{2}}\right) \Psi_{D}^{s_{D}}\left(p_{i_{1}}, s_{i_{1}} ; p_{s_{1}}, s_{s}\right)}{2 \sqrt{E_{s_{2}} E_{s_{1}}}} \\
& \times\left\langle p_{X_{2}}, s_{X_{2}} ; p_{s_{2}}, s_{s_{2}}\left|F_{N X_{1}, N X_{2}}^{(\mathrm{off})}\right| p_{X_{1}}, s_{X_{1}} ; p_{s_{1}}, s_{S_{1}}\right\rangle J_{\gamma N X_{2}}^{\mu \dagger}\left(p_{i_{2}}, s_{i_{2}} ; p_{X_{2}}, s_{X_{2}}\right) J_{\gamma N X_{1}}^{v}\left(p_{i_{1}}, s_{i_{1}} ; p_{X_{1}}, s_{X_{1}}\right) \frac{1}{p_{X_{1}}^{2}-m_{X_{1}}^{2}} \frac{1}{p_{X_{2}}^{2}-m_{X_{2}}^{2}}
\end{aligned}
$$

where $\int_{\mathcal{P}}$ indicates Cauchy principal value integration. The total FSI contribution to the deuteron hadronic tensor is then given by the sum $W_{\mathrm{FSI}}^{\mu \nu}=W_{\mathrm{FSI}}^{\mu \nu \text { (on) }}+W_{\mathrm{FSI}}^{\mu \nu(\text { off })}$. The results in Eqs. (24) and (25) form the general expression for the FSI contribution to the deuteron hadronic tensor within the eikonal approximation. To evaluate these expressions in practice requires modeling of the matrix elements of $F_{N X_{1}, N X_{2}}$ and a truncation of the set of states comprising the inelastic intermediate states in Fig. 2, to which we turn in the next section.

\section{FACTORIZED EFFECTIVE RESONANCE MODEL}

Considerable experience has been developed over the years with the computation of the Born approximation contribution [Eq. (19)] to the deuteron hadronic tensor, especially since the observation of the nuclear EMC effect, or deviation from unity of the ratio of nuclear to deuterium structure functions, some three decades ago [48]. For the deuteron, the input required in these calculations is the nucleon momentum distribution in the deuteron and the bound nucleon structure functions in the nucleon hadronic tensor $W_{N}^{\mu \nu}$. In the VNA the latter is approximated by the on-shell hadronic tensor, with the off-shell nucleon momentum defined through the on-shell momentum of the deuteron and spectator nucleon. Some attempts have been made, however, to account for the possible effects on the bound nucleon structure of nuclear medium modification, or off-shell corrections (see, e.g., Refs. [7,13,18,44,49-52].

Much less is understood about the FSI corrections [Eqs. (24) and (25)], however, which require knowledge of the inelastic currents and rescattering amplitude for the interaction between the hadronic debris of the (off-shell) scattered nucleon and the (on-shell) spectator nucleon. The complexity of describing this interaction is formidable, however, and as an exploratory attempt to estimate the FSI effects numerically we use several additional assumptions to make the calculation feasible.

\section{A. Factorized approximation for FSI}

In the factorized approximation [also referred to as the distorted wave impulse approximation (DWIA)], the inelastic electromagnetic currents in both Eqs. (24) and (25) are diagonalized by factoring out the current $J_{\gamma N X_{2}}^{\mu \dagger}$ from the $d^{3} p_{s_{2}}$ integration evaluating it at $p_{i_{2}}=p_{i_{1}}, s_{i_{2}}=s_{i_{1}}, p_{X_{2}}=p_{X_{1}}$, and $s_{X_{2}}=s_{X_{1}}$ (and hence $m_{X_{1}} \approx m_{X_{2}}$ ). This approximation is well known in quasielastic nuclear processes, where it allows meaningful bound nucleon electromagnetic structure functions to be identified when considering FSI effects (see, e.g., Ref. [53]). In the following, we proceed with the DWIA derivations of on-shell [Eq. (24)] and off-shell [Eq. (25)] hadronic tensors separately. We note also that the factorization approximation breaks the explicit symmetry of Eqs. (24) and (25) with respect to the " 1 " and " 2 " indices. However, in numerical calculations this symmetry can be restored by taking the average of results for the above factorization and one following from the factorization of the $J_{\gamma N X_{1}}^{v}$ current from the $d^{3} p_{s_{2}}$ integration.

For the on-shell hadronic tensor we first express the $X_{1} N \rightarrow X_{2} N$ invariant scattering amplitude through the diffractive amplitude, imposing the condition for helicity conservation in the form

$$
\begin{aligned}
& \left\langle p_{X_{2}}, s_{X_{2}} ; p_{s_{2}}, s_{s_{2}}\left|F_{N X_{1}, N X_{2}}\left(s_{X N}, t_{X N}\right)\right| p_{X_{1}}, s_{X_{1}} ; p_{s_{1}}, s_{S_{1}}\right\rangle \\
& =\eta\left(s_{X N}, m_{X_{1}}\right)\left\langle p_{X_{2}}, p_{s_{2}}\left|f_{N X_{1}, N X_{2}}\left(s_{X N}, t_{X N}\right)\right| p_{X_{1}}, p_{s_{1}}\right\rangle \\
& \quad \times \delta_{s_{s_{1}}, s_{s_{2}}} \delta_{S_{X_{1}}, s_{X_{2}}},
\end{aligned}
$$

where $\eta\left(s_{X N}, m_{X_{1}}\right)=\sqrt{\left[s_{X_{N}}-\left(m-m_{X_{1}}\right)^{2}\right]\left[s_{X_{N}}-\left(m+m_{X_{1}}\right)^{2}\right]}$, with the Mandelstam variables $s_{X N}=\left(p_{X_{1}}+p_{s_{1}}\right)^{2}=\left(p_{X_{2}}+p_{s_{2}}\right)^{2}$ 
and $t_{X N}=\left(p_{X_{1}}-p_{X_{2}}\right)^{2}=\left(p_{s_{1}}-p_{s_{2}}\right)^{2}$. The amplitude $f_{N X_{1}, N X_{2}}$ is defined in the eikonal approximation,

$$
f_{N X_{1}, N X_{2}}\left(t_{X N}\right)=\sigma_{\text {tot }}(i+\epsilon) \exp \left(\beta t_{X N} / 2\right)
$$

where $\sigma_{\text {tot }}$ represents the total cross section of the scattering of the produced $X^{\prime}$ system from the spectator nucleon, $\beta$ is the slope factor, and $\epsilon$ represents the ratio of the real to imaginary parts of the amplitude.

The approximation of helicity conservation allows the spin summations in the electromagnetic current to be factorized from the FSI. Combining then the factorized current $J_{\gamma N X_{1}}^{\mu \dagger}$ with $J_{\gamma N X_{1}}^{\nu}$ and $\delta\left(p_{X_{1}}^{2}-m_{X_{1}}^{2}\right)$, one obtains the hadronic tensor of the bound nucleon $W_{N}^{\mu \nu}\left(p_{i_{1}}, Q^{2}, m_{X_{1}}\right)$ using Eq. (18). In the DWIA this tensor is defined by the same kinematical conditions as the tensor in the Born term of Eq. (19), with the invariant mass $m_{X_{1}}^{2}=\left(p_{D}-p_{s_{1}}+q\right)^{2}$ determined through the integration over the spectator momentum $\boldsymbol{p}_{s_{1}}$, and the momentum fraction of the initial struck nucleon is

$$
x_{1}=\frac{Q^{2}}{2 p_{i_{1}} \cdot q}=\frac{m x}{M_{D}-E_{s_{1}}+p_{s_{1}, z}|q| / q^{0}}<1 .
$$

Note that even though the kinematics entering in $W_{N}^{\mu \nu}\left(p_{i_{1}}, Q^{2}, m_{X_{1}}\right)$ are the same as for the Born contribution, we still retain the subscripts " 1 " to distinguish these from the kinematics after rescattering.

For the rescattering part of Eq. (24), the integration region is restricted by the condition on the momentum fraction of the final nucleon $p_{i_{2}}$,

$$
x_{2}=\frac{Q^{2}}{2 p_{i_{2}} \cdot q}<1 .
$$

The $p_{s_{2}, z}$ integration in Eq. (24) is taken using the remaining $\delta$ function,

$$
\delta\left(p_{X_{2}}^{2}-m_{X_{2}}^{2}\right)=\frac{1}{2}|| \boldsymbol{q}\left|-\left(M_{D}+q^{0}\right) \frac{\widetilde{p}_{s_{2}, z}^{X_{2}}}{\widetilde{E}_{s_{2}}}\right|^{-1} \delta\left(p_{s_{2}, z}-\widetilde{p}_{s_{2}, z}^{X_{2}}\right),
$$

where $\widetilde{E}_{s_{2}}=\sqrt{m^{2}+p_{s_{2}, \perp}^{2}+\left(\widetilde{p}_{s_{2}, z}^{X_{2}}\right)^{2}}$, and $\widetilde{p}_{s_{2}, z}^{X_{2}}$ is obtained from the solution of

$$
\begin{aligned}
& 2|\boldsymbol{q}| \widetilde{p}_{s_{2}, z}^{X_{2}}-2\left(M_{D}+q^{0}\right) \widetilde{E}_{S_{2}} \\
& \quad=m_{X_{2}}^{2}-M_{D}^{2}+Q^{2}-2 M_{D} q^{0}-m^{2} .
\end{aligned}
$$

The FSI part of the structure function will then be defined by the integration of the transverse component $\boldsymbol{p}_{s_{2}, \perp}$ of the spectator momentum. Combining the factorized inelastic nucleon structure function with the on-shell part of the FSI term of Eq. (24), one finally obtains for the on-shell FSI contribution

$$
\begin{aligned}
W_{\mathrm{FSI}}^{\mu \nu \text { (on) }}= & -\sum_{N} \frac{2 m}{M_{D}} \int d^{3} \boldsymbol{p}_{s_{1}} \frac{W_{N}^{\mu \nu}\left(p_{i_{1}}, q, m_{X_{1}}\right)}{8 \sqrt{E_{s_{1}}}} \frac{1}{3} \sum_{X_{2}} \sum_{s_{i}, s_{s}, s_{D}} \int \frac{d^{2} \boldsymbol{p}_{s_{2}, \perp}}{(2 \pi)^{2}} \\
& \times \operatorname{Im}\left\{\frac{\eta\left(s_{X N}, m_{X_{1}}\right) f_{N\left\{X_{1}\right\}, N X_{2}}^{(\mathrm{on})}\left(t_{X N}\right)}{|| \boldsymbol{q}\left|-\left(M_{D}+q^{0}\right) \widetilde{p}_{s_{2}, z}^{X_{2}} / \widetilde{E}_{s_{2}}\right| \sqrt{\widetilde{E}_{S_{2}}}} C\left(p_{i_{1}}, \widetilde{p}_{i_{2}}^{X_{2}}, q\right) \Psi_{D}^{s_{D} \dagger}\left(\widetilde{p}_{i_{2}}^{X_{2}}, s_{i} ; \widetilde{p}_{s_{2}}^{X_{2}}, s_{s}\right) \Psi_{D}^{s_{D}}\left(p_{i_{1}}, s_{i} ; p_{s_{1}}, s_{s}\right)\right\},
\end{aligned}
$$

where the four-vectors $\widetilde{p}_{s_{2}}^{X_{2}}=\left(\widetilde{E}_{s_{2}} ; \boldsymbol{p}_{s_{2}, \perp}, \widetilde{p}_{s_{2}, z}^{X_{2}}\right)$ and $\widetilde{p}_{i_{2}}^{X_{2}}=p_{D}-\widetilde{p}_{s_{2}}^{X_{2}}$, with $\left\{X_{1}\right\}$ denoting intermediate states that obey $p_{X_{1}}^{2}=$ $m_{X_{1}}^{2}$, and we use the shorthand notation $s_{i} \equiv s_{i_{1}}=s_{i_{2}}$ and $s_{s} \equiv s_{s_{1}}=s_{s_{2}}$. The symmetrization factor $C\left(p_{i_{1}}, \widetilde{p}_{i_{2}}^{X_{2}}, q\right)$ accounts for the choice of momenta at which the currents are evaluated in the factorization approximation (see Sec. IV below).

Using the solution of Eq. (31) one obtains the more explicit expression for the momentum fraction $x_{2}$ entering in the FSI part of Eq. (24),

$$
x_{2}=\frac{1}{1+\left[m_{X_{2}}^{2}-\left(\tilde{p}_{i_{2}}^{X_{2}}\right)^{2}\right] / Q^{2}} \approx \frac{1}{1+\left(m_{X_{2}}^{2}-m^{2}\right) / Q^{2}} .
$$

The important feature of this expression is that it shows that for any fixed value of $m_{X_{2}}$, the FSI terms are suppressed kinematically in the Bjorken limit, where $x_{2} \rightarrow 1$.

For the off-shell part of the FSI amplitude of Eq. (25) one performs a similar factorization of the electromagnetic currents, using helicity conservation in the rescattering amplitude [as in Eq. (26)]. The principal value integrations can be performed in Eq. (25) employing the method outlined in Ref. [32], and using the analytic structure of the deuteron wave function one obtains

$$
\int_{\mathcal{P}} d p_{s, z} \frac{\Psi_{D}^{s_{D}}\left(p_{i}, s_{i} ; p_{s}, s_{s}\right)}{p_{s, z}-\widetilde{p}_{s, z}^{X}}=-\pi \widetilde{p}_{s, z}^{X} \widetilde{\Psi}_{D}^{s_{D}}\left(\widetilde{p}_{i}^{X}, s_{i} ; \widetilde{p}_{s}^{X}, s_{s}\right)
$$

with $\widetilde{\Psi}_{D}^{s_{D}}$ representing the distorted wave function of the deuteron whose explicit form is given in Ref. [32]. After performing the above factorization and the principal value integrations over $d p_{s_{1}}^{z}$ and $d p_{s_{2}}^{z}$ for the off-shell part of the FSI of Eq. (25), 
one finds

$$
\begin{aligned}
W_{\mathrm{FSI}}^{\mu \nu \text { (off) }}= & \frac{1}{6 M_{D}} \sum_{N, X_{1}, X_{2}} \sum_{s_{i}, s_{s}, s_{D}} \int d^{2} \boldsymbol{p}_{s_{1, \perp}} \widetilde{p}_{s_{1, z}}^{X_{1}} \frac{J_{\gamma N X_{1}}^{\mu \dagger}\left(\widetilde{p}_{i_{1}}^{X_{1}}, s_{i} ; p_{X_{1}}, s_{X}\right) J_{\gamma N X_{1}}^{v}\left(\widetilde{p}_{i_{1}}^{X_{1}}, s_{i} ; p_{X_{1}}, s_{X}\right)}{8|| \boldsymbol{q}\left|-\left(M_{D}+q^{0}\right) \widetilde{p}_{s_{1}, z}^{X_{1}} / \widetilde{E}_{s_{1}}\right| \sqrt{\widetilde{E}_{s_{1}}}} \\
& \times \int \frac{d^{2} \boldsymbol{p}_{s_{2, \perp}}}{(2 \pi)^{2}} \widetilde{p}_{s_{2, z}}^{X_{2}} \operatorname{Im}\left\{\frac{\eta\left(s_{X N}, m_{X_{1}}\right) f_{N X_{1}, N X_{2}}^{\text {(off) }}\left(t_{X N}\right)}{|| \boldsymbol{q}\left|-\left(M_{D}+q^{0}\right) \widetilde{p}_{s_{2, z}}^{X_{2}} / \widetilde{E}_{s_{2}}\right| \sqrt{\widetilde{E}_{s_{2}}}} \widetilde{\Psi}_{D}^{s_{D} \dagger}\left(\widetilde{p}_{i_{1}}^{X_{1}}, s_{i} ; \widetilde{p}_{s_{1}}^{X_{1}}, s_{s}\right) \widetilde{\Psi}_{D}^{s_{D}}\left(\widetilde{p}_{i_{2}}^{X_{2}}, s_{i} ; \widetilde{p}_{s_{2}}^{X_{2}}, s_{s}\right)\right\} .
\end{aligned}
$$

However, to introduce an off-shell nucleon hadronic tensor $W_{N}^{\mu \nu \text { (off) }}$ one needs to add an additional integral,

$$
1=\int d W^{2} \delta\left(p_{X_{1}}^{2}-W^{2}\right)
$$

that allows the inelastic electromagnetic currents to be combined to form the hadronic tensor of the nucleon in the form of Eq. (18). Finally, we obtain for the off-shell tensor

$$
\begin{aligned}
& W_{\mathrm{FSI}}^{\mu \nu \text { (off) }}=\sum_{N, X_{2}} \frac{2 m}{M_{D}} \int d W W \int d^{2} \boldsymbol{p}_{s_{1, \perp}} \widetilde{p}_{s_{1, z}}^{X_{2}} \frac{W_{N}^{\mu \nu(\text { off })}\left(\tilde{p}_{i_{1}}^{X_{2}}, q, W\right)}{8|| \boldsymbol{q}\left|-\left(M_{D}+q^{0}\right) \widetilde{p}_{s_{1}, z}^{X_{2}} / \widetilde{E}_{s_{1}}\right| \sqrt{\widetilde{E}_{s_{1}}}} \frac{1}{3} \sum_{s_{i}, s_{s}, s_{D}} \int \frac{d^{2} \boldsymbol{p}_{s_{2, \perp}}}{(2 \pi)^{2}} \tilde{p}_{s_{2, z}}^{X_{2}} \\
& \times \operatorname{Im}\left\{\frac{\eta\left(s_{X N}, W\right) f_{N\left\{X_{1}\right\}_{W}, N X_{2}}^{(\text {off }}\left(t_{X N}\right)}{|| \boldsymbol{q}\left|-\left(M_{D}+q^{0}\right) \widetilde{p}_{s_{2, z}}^{X_{2}} / \widetilde{E}_{s_{2}}\right| \sqrt{\widetilde{E}_{s_{2}}}} C\left(\widetilde{p}_{i_{1}}^{X_{2}}, \widetilde{p}_{i_{2}}^{X_{2}}, q\right) \widetilde{\Psi}_{D}^{s_{D} \dagger}\left(\widetilde{p}_{i_{1}}^{X_{2}}, s_{i} ; \widetilde{p}_{s_{1}}^{X_{2}}, s_{s}\right) \widetilde{\Psi}_{D}^{s_{D}}\left(\widetilde{p}_{i_{2}}^{X_{2}}, s_{i} ; \widetilde{p}_{s_{2}}^{X_{2}}, s_{s}\right)\right\},
\end{aligned}
$$

where $\left\{X_{1}\right\}_{W}$ here are intermediate states with $p_{X_{1}}^{2}=W^{2}$. Because the summation over $X_{1}$ is absorbed in the definition of the nucleon hadronic tensor in Eq. (18), the longitudinal component of the initial spectator nucleon momentum $\widetilde{p}_{s_{1}, z}^{X_{2}}$ is evaluated at $m_{X_{2}}$ in Eq. (31), because this will minimize $t_{X N}$ $\left(\widetilde{p}_{s_{1}, z}^{X_{2}} \approx \widetilde{p}_{s_{2}, z}^{X_{2}}\right)$. This maximizes the eikonal rescattering amplitude $f_{N\left\{X_{1}\right\}_{W}, N X_{2}}^{(\text {off }}\left(t_{X N}\right)$, allowing us to provide an estimate for the maximum possible effect of FSI.

Notice that in Eq. (37) the nucleon hadronic tensor and rescattering amplitude are computed at the Bjorken scaling variables $x_{1}$ and $x_{2}$, respectively. They can be calculated using the identity in Eq. (36) and the property of the principal value integration in Eq. (34), which defines the longitudinal component $p_{i_{2}, z}$ through energy-momentum conservation [Eq. (31)]. This yields the approximate expressions

$x_{1} \approx \frac{1}{1+\left(W^{2}-m^{2}\right) / Q^{2}} \quad$ and $\quad x_{2} \approx \frac{1}{1+\left(m_{X_{2}}^{2}-m^{2}\right) / Q^{2}}$,

where $W$ is the mass over which the bound nucleon hadronic tensor is integrated, and $m_{X_{2}}$ is the mass of the intermediate inelastic state produced in the rescattering. Similar to the onshell case, here too one finds that at large $Q^{2}$ both $x_{1}$ and $x_{2} \rightarrow 1$, suppressing the FSI contribution. We note that the disappearance of the FSIs in the Bjorken limit is independent of the factorization approximation, because, in general, the values of $x_{1}$ and $x_{2}$ are defined by $m_{X_{1}}$ and $m_{X_{2}}$ [similar to Eq. (38)]. The observation that in the Bjorken limit the FSIs disappear follows therefore from the general property of the eikonal approximation which assumes a rescattering between effective hadronic states with masses $m_{X_{1}}$ and $m_{X_{2}}$.

\section{B. Constraints from SIDIS}

In the earlier studies of FSI effects in SIDIS [32], the mass produced in the intermediate state by the virtual photon scattering from the moving nucleon was fixed by the kinematics of the detected spectator nucleon. In the inclusive DIS case, however, the sum must be taken in Eqs. (32) and (37) over all possible intermediate inelastic states produced at the $\gamma^{*} N X$ vertex that can rescatter from the spectator nucleon.

Inclusion of the complete set of states in Fig. 2(b) that can contribute to the rescattering is, of course, not feasible, and, in practice, a truncation of the spectrum is necessary, which inevitably introduces an element of model dependence into the calculation. From measurements of nucleon inelastic structure functions at intermediate values of $Q^{2}$, three clear resonance structures are seen to dominate the spectrum in the low- $W$ region, as illustrated in Fig. 4, centered at masses $W_{\text {res }}=1.232,1.5$, and $1.75 \mathrm{GeV}$. In the present model we do not account for any relative phases between the amplitudes for the different resonance contributions, so that our estimate provides an upper bound on the size of the FSI effects in this kinematic region. From Fig. 3 it is clear that for $W_{\text {res }}<$ $1.75 \mathrm{GeV}$ the value of $x_{\lim }$ remains unity for $Q^{2}$ as low as $4 \mathrm{GeV}^{2}$ and is still reasonably high $\left(x_{\lim } \gtrsim 0.7\right)$ for $Q^{2}=2$ $\mathrm{GeV}^{2}$, suggesting that the eikonal approximation for the threeresonance model should be valid down to these $Q^{2}$ values.

For larger invariant masses, above the resonance region, the analysis [32] of deuteron SIDIS data [34] suggests that FSIs yield sizeable contributions at the highest measured $W$ bins ( $W=2.0$ and $2.4 \mathrm{GeV}$ ) and should therefore also be included in our numerical estimates. As discussed in Sec. I, however, for large invariant masses the phase space for the intermediate state becomes unrestricted and the closure relation is expected to hold in inclusive scattering. To include contributions from 


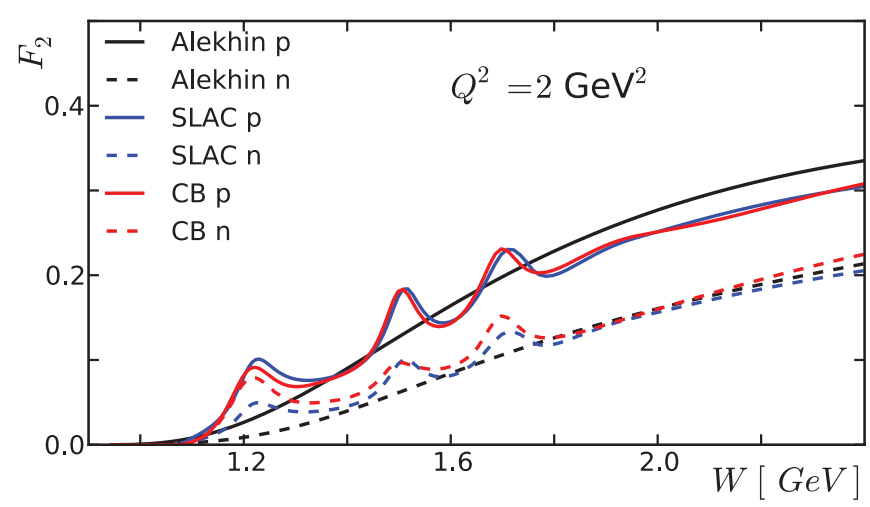

FIG. 4. (Color online) $F_{2}$ proton (solid lines) and neutron (dashed lines) structure functions at $Q^{2}=2 \mathrm{GeV}^{2}$ for the Alekhin leading twist [54] (black), SLAC [55] (blue), and Christy and Bosted (CB) [56] (red) parametrizations of $F_{2}$.

the $W \gtrsim 2 \mathrm{GeV}$ region, where no clear resonance structure is visible, we consider specific widths in $W$ and fold the FSI contributions of Eqs. (32) and (37) with a distribution in $W$, normalized to the values extracted from the analysis of the SIDIS data [32]. This simple approximation allows the effects of FSIs to be estimated at small $x$ values, in addition to those at large $x$ which are determined by the resonance contributions. Note, however, that care must be taken when including contributions with high $W(W \gtrsim 3.5 \mathrm{GeV})$, as at low $Q^{2}$ values the intermediate states have momenta below the limit where the eikonal approximation is known to be reliable.

The overall FSI contribution to the deuteron structure function is therefore obtained by computing the total FSI hadronic tensor,

$$
\begin{aligned}
W_{\mathrm{FSI}}^{\mu \nu(\mathrm{tot})}= & \int d m_{X_{2}}\left[\sum_{\text {res }} \delta\left(m_{X_{2}}-W_{\mathrm{res}}\right)+\rho\left(m_{X_{2}}\right)\right] \\
& \times\left(W_{\mathrm{FSI}}^{\mu \nu(\text { on })}+W_{\mathrm{FSI}}^{\mu \nu(\mathrm{off})}\right),
\end{aligned}
$$

where the sum runs over the above-mentioned resonances and $\rho\left(m_{X_{2}}\right)$ denotes the spectral function representing the DIS continuum region $(W>2 \mathrm{GeV})$. In the following section we present numerical estimates for the FSI effects on the inclusive deuteron structure functions.

\section{RESULTS}

In this section we examine the effects of including the FSI contribution to the inclusive DIS cross section of the deuteron using the approach outlined in Sec. III. We present calculations for the inclusive structure function $F_{2}^{D}$, which is related to the semi-inclusive structure functions $F_{L}^{D}$ and $F_{T}^{D}$ discussed in Ref. [32],

$F_{2}^{D}=\sum_{N} \int \frac{d^{3} \boldsymbol{p}_{s}}{(2 \pi)^{2} 2 E_{s}}\left[F_{L}^{D}\left(Q^{2}, \tilde{x}, \boldsymbol{p}_{s}\right)+\frac{x}{\gamma^{2}} F_{T}^{D}\left(Q^{2}, \tilde{x}, \boldsymbol{p}_{s}\right)\right]$,

where $\gamma^{2} \equiv \boldsymbol{q}^{2} / q_{0}^{2}=1+4 x^{2} m^{2} / Q^{2}$, and the variable $\tilde{x}$ is defined according to Eq. (28). Unless noted otherwise, we use the SLAC parametrization [55] for the proton and neutron

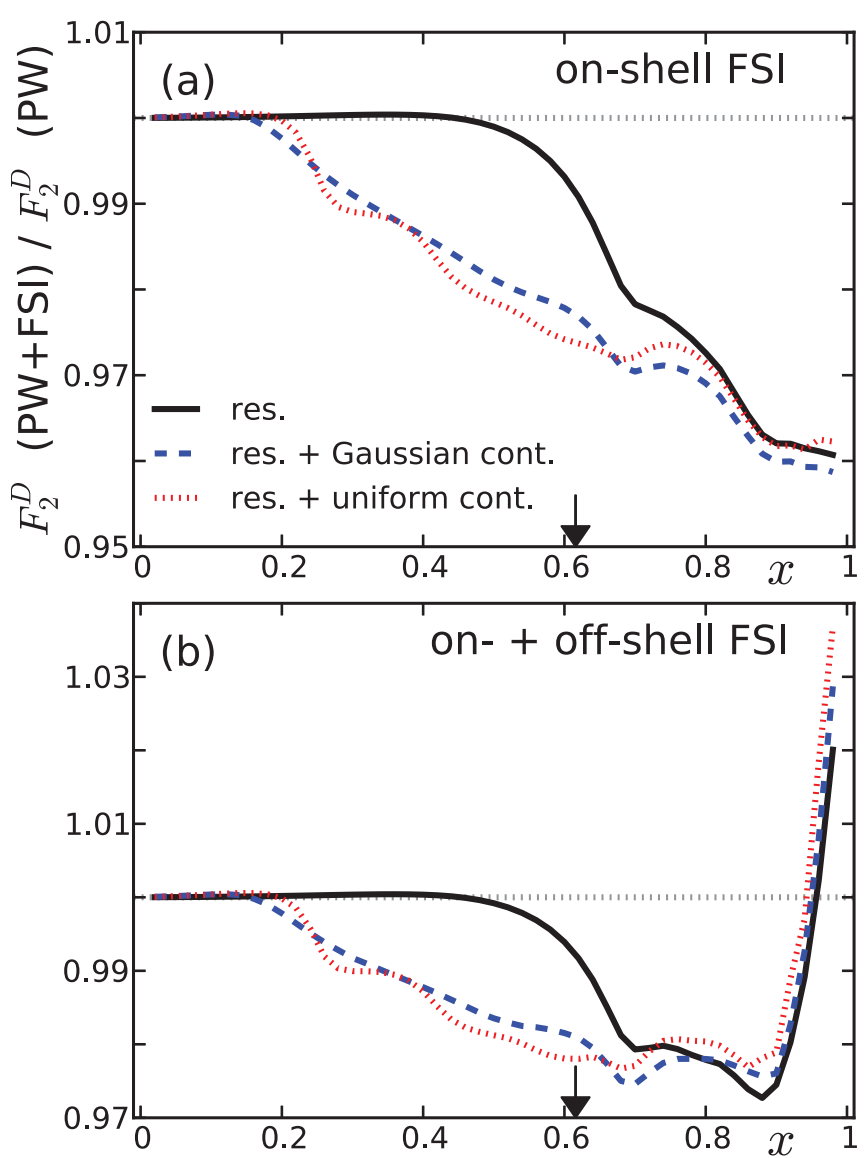

FIG. 5. (Color online) Ratio of the deuteron $F_{2}^{D}$ structure function with FSIs to that computed in the plane-wave (PW) approximation at $Q^{2}=5 \mathrm{GeV}^{2}$, using only the on-shell amplitude in Eq. (32) (a) and including also the off-shell contribution of Eq. (37) (b). The results with the resonance region contributions alone (black solid lines) are compared with those including a continuum component with a Gaussian distribution (blue dashed lines) and a uniform distribution (red dotted lines). The arrow along the $x$ axis indicates the boundary at $W=2 \mathrm{GeV}$ between the resonance and DIS regions for free nucleon kinematics.

structure functions, which covers a large range of $W$ and $Q^{2}$, including the nucleon resonance structure. To parametrize the $W$ and $Q^{2}$ dependence of the rescattering parameters entering in Eq. (27), we use the results of the analysis [32] of the semi-inclusive DIS data from Ref. [34]. For the ratio of the real to imaginary parts of the cross section and the slope factor, we take fixed values $\epsilon=-0.2$ and $\beta=8 \mathrm{GeV}^{2}$, respectively. In the region of $W$ between the $\Delta(1232)$ resonance and the highest mass accessed in the experiment [34], $W=W_{0} \equiv 2.4$ $\mathrm{GeV}$, the extracted cross section rises linearly with $W$ while falling with $Q^{2}$,

$$
\sigma_{\text {tot }}\left(W, Q^{2}\right)=\frac{a+b W}{Q^{2}},
$$

with the constants $a=25.3 \mathrm{mb}$ and $b=53 \mathrm{mb} / \mathrm{GeV}$ fitted to the analysis of Ref. [32]. At higher $W$, where the DIS cross section exhibits scaling, we take $\sigma_{\text {tot }}\left(W>W_{0}, Q^{2}\right)=$ $\sigma_{\text {tot }}\left(W_{0}, Q^{2}\right)$. To further account for the off-shell nature of 

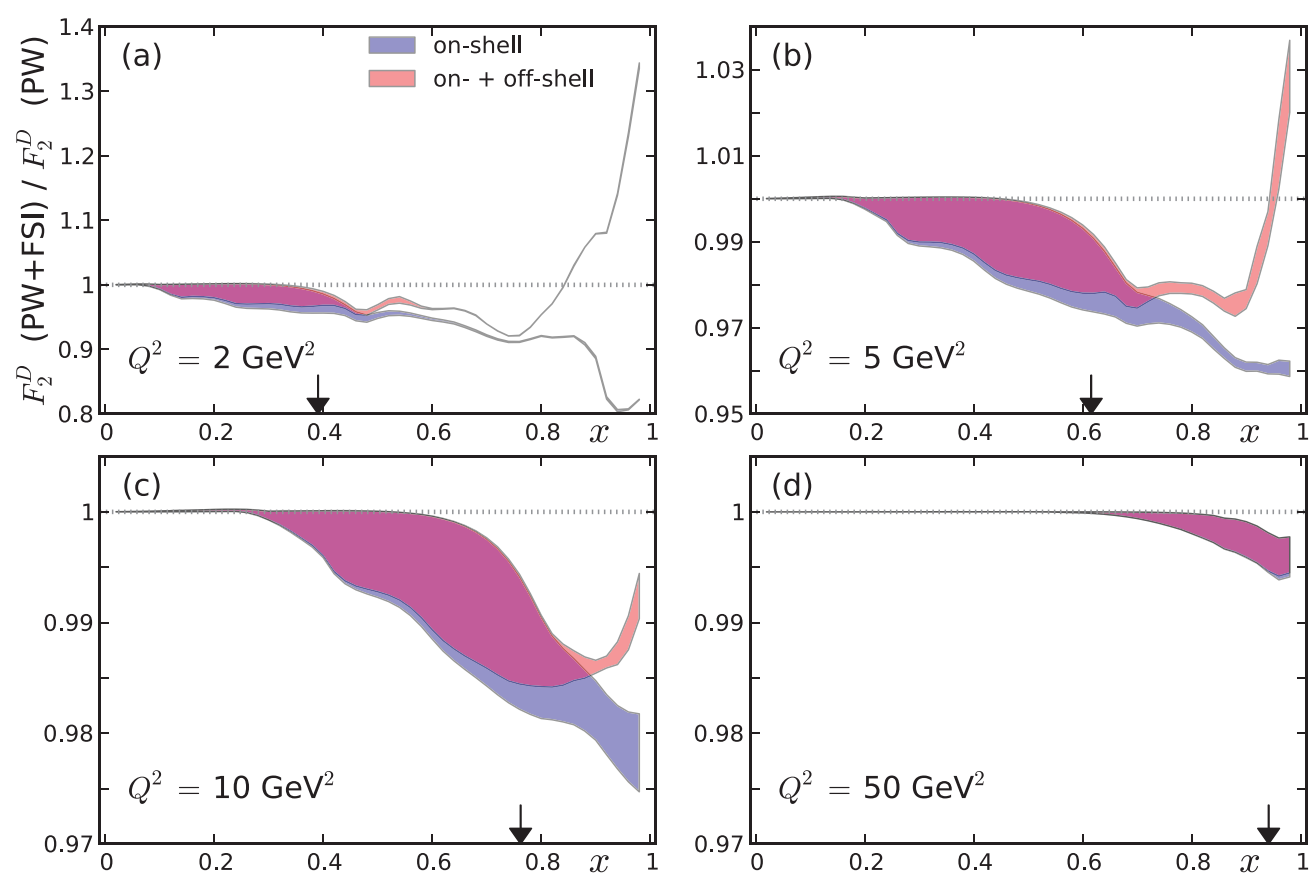

FIG. 6. (Color online) Ratio of the deuteron $F_{2}^{D}$ structure function including FSIs to that computed in the PW approximation, at several values of $Q^{2}$ from 2 to $50 \mathrm{GeV}^{2}$. The on shell only results from Eq. (32) (blue shaded bands) and those including off-shell contributions from Eq. (37) (orange shaded bands) span the range of models for the distribution of intermediate-state masses shown in Fig. 5. The arrows along the $x$ axis indicate the $W=2 \mathrm{GeV}$ point at each $Q^{2}$ value for free nucleon kinematics.

the inelastic intermediate states in the hadronic currents and rescattering amplitude in Eq. (37), we introduce a suppression factor [40] for the off-shell amplitude,

$$
f_{N X_{1}, N X_{2}}^{\text {(off) }}\left(t_{X N}\right)=f_{N X_{1}, N X_{2}}^{(\text {on })}\left(t_{X N}\right) \exp \left(\beta\left|W^{2}-m_{X_{2}}^{2}\right| / 2\right),
$$

where $W$ is the invariant mass that enters in the nucleon hadronic tensor. For the nonrelativistic deuteron wave function we use the parametrization based on the Paris $N N$ potential [57], but also compare the results with the CD-Bonn [58] and WCJ-1 [59] wave functions. The symmetrization factor $C\left(p_{i_{1}}, p_{i_{2}}, q\right)$ introduced in Eqs. (32) and (37) is taken as

$$
C\left(p_{i_{1}}, p_{i_{2}}, q\right)=\sqrt{\frac{F_{2}^{N}\left(x_{2}, Q^{2}\right)}{F_{2}^{N}\left(x_{1}, Q^{2}\right)},}
$$

where $F_{2}^{N}$ is the nucleon structure function and ensures that the kinematical constraints $x_{1}, x_{2}<1$ are taken into account.

The ratio of the $F_{2}^{D}$ structure function evaluated including the effects of FSIs to that with the pw contribution only is illustrated in Fig. 5 at $Q^{2}=5 \mathrm{GeV}^{2}$. The results with the three resonance contributions alone are compared with those that account also for the DIS continuum. For the shape of the continuum spectral function $\rho\left(m_{X_{2}}\right)$ in Eq. (39) we consider two different parametrizations, based on Gaussian and uniform distributions. The Gaussian distributions are chosen to be centered at $m_{X_{2}}=\{2,2.5,3.4\} \mathrm{GeV}$, with corresponding widths of $\{300,500,700\} \mathrm{MeV}$, to correspond to the values of the two highest $W$ bins in Ref. [34]. The uniform parametrization uses three distributions limited by
$m_{X_{2}}$ values of $\{1.85,2.2,2.8,4\} \mathrm{GeV}$. While the two $\rho\left(m_{X_{2}}\right)$ models exhibit quite different shapes, the difference between them has only a very modest effect on the $F_{2}^{D}$ ratios in Fig. 5.

The inclusion of the FSI contribution generally suppresses the $F_{2}^{D}$ structure function by several percent, particularly at high values of $x$, where the intermediate states have greatest phase space available. With the resonance contributions only, and for the on-shell rescattering amplitude, the structure function is reduced by $\approx 1 \%-2 \%$ for $x \approx 0.6-0.7$ and by up to $\approx 3 \%-4 \%$ for $x \gtrsim 0.8$. The effect of the continuum contribution is to reduce $F_{2}^{D}$ at lower values of $x$ for masses below the free nucleon threshold of $W=2 \mathrm{GeV}$ (corresponding to $x \lesssim 0.6$ at $Q^{2}=5 \mathrm{GeV}^{2}$ ). The addition of the off-shell FSI scattering amplitude in Eq. (42) curtails some of the $F_{2}^{D}$ suppression, to $\approx 2 \%$ up to $x \approx 0.9$, with a strong enhancement of the ratio above unity for $x \rightarrow 1$, which is largely independent of the details of the distribution of the intermediate-state masses.

The $Q^{2}$ dependence of the FSI contributions is illustrated in Fig. 6 for $F_{2}^{D}$ calculated in terms of on-shell-only amplitudes and including the off-shell corrections. Here the bands envelop the range of intermediate-state mass distributions considered in Fig. 5, including the resonance components and the models for the DIS continuum. For the on-shell part of the FSI amplitude in Eq. (32) the largest effect is seen at the lowest $Q^{2}$ values, where the invariant masses of the resonances contributing to the FSI amplitude are attained for $x \approx 0.4-1$ and the $Q^{2}$ suppression of the rescattering amplitude is weakest. The $F_{D}^{2}$ structure function ratio in this case is below unity for all values of $x$, with the largest effect seen at $x \gtrsim 0.9$, where $F_{2}^{D}$ is 

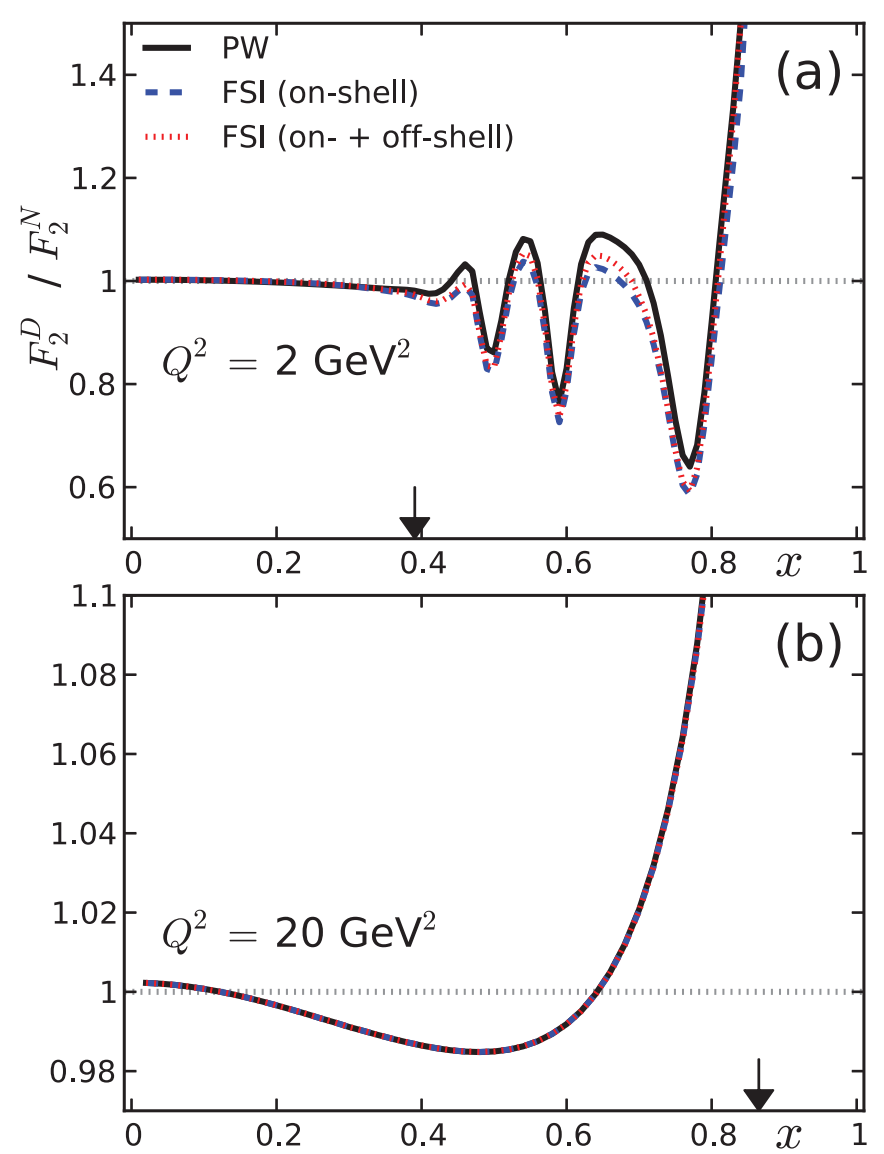

FIG. 7. (Color online) Ratio of the deuteron $F_{2}^{D}$ to isoscalar nucleon $F_{2}^{N}$ structure functions at $Q^{2}=2 \mathrm{GeV}^{2}$ (a) and $Q^{2}=20 \mathrm{GeV}^{2}$ (b). The PW results (black solid curves) are compared with those including FSIs with the on-shell amplitude [Eq. (32)] (blue dashed curves) and with the addition of the off-shell contribution of [Eq. (37)] (red dotted curves). The arrows along the $x$ axis indicate the $W=2$ $\mathrm{GeV}$ boundary for free nucleon kinematics. The nucleon resonance structures are clearly visible in the ratio at $Q^{2}=2 \mathrm{GeV}^{2}$.

reduced by up to $20 \%$ at $Q^{2}=2 \mathrm{GeV}^{2}$. At larger $Q^{2}$, the effect is significantly smaller, with $F_{2}^{D}$ suppressed by less than $0.5 \%$ at $Q^{2}=50 \mathrm{GeV}^{2}$.

The contribution of the off-shell amplitude has the opposite effect on $F_{2}^{D}$ to that of the on-shell amplitude. The size of the off-shell contribution is negligible at low $x$, but becomes important at high $x$ and low $Q^{2}$. This behavior can be expected if one considers a dynamical picture based on the analysis of the propagation of the virtual particle in the intermediate state of the reaction [60]. As with the on-shell term, the size of the off-shell contribution becomes smaller with increasing $Q^{2}$, reflecting the suppression of FSI effects at large $Q^{2}$. In the high- $x$ region all possible $m_{X_{2}}$ contributions included in Eq. (39) are kinematically accessible for the phase space of the produced $X$, so that all possible rescattering contributions can play a role. We should stress, however, that (as noted above) the curves in Fig. 6 represent the maximum possible effect of the FSI contribution in our model.

To put the effects of the FSIs in a more familiar context, in Fig. 7 we show the ratio of the deuteron $F_{2}^{D}$ to isoscalar
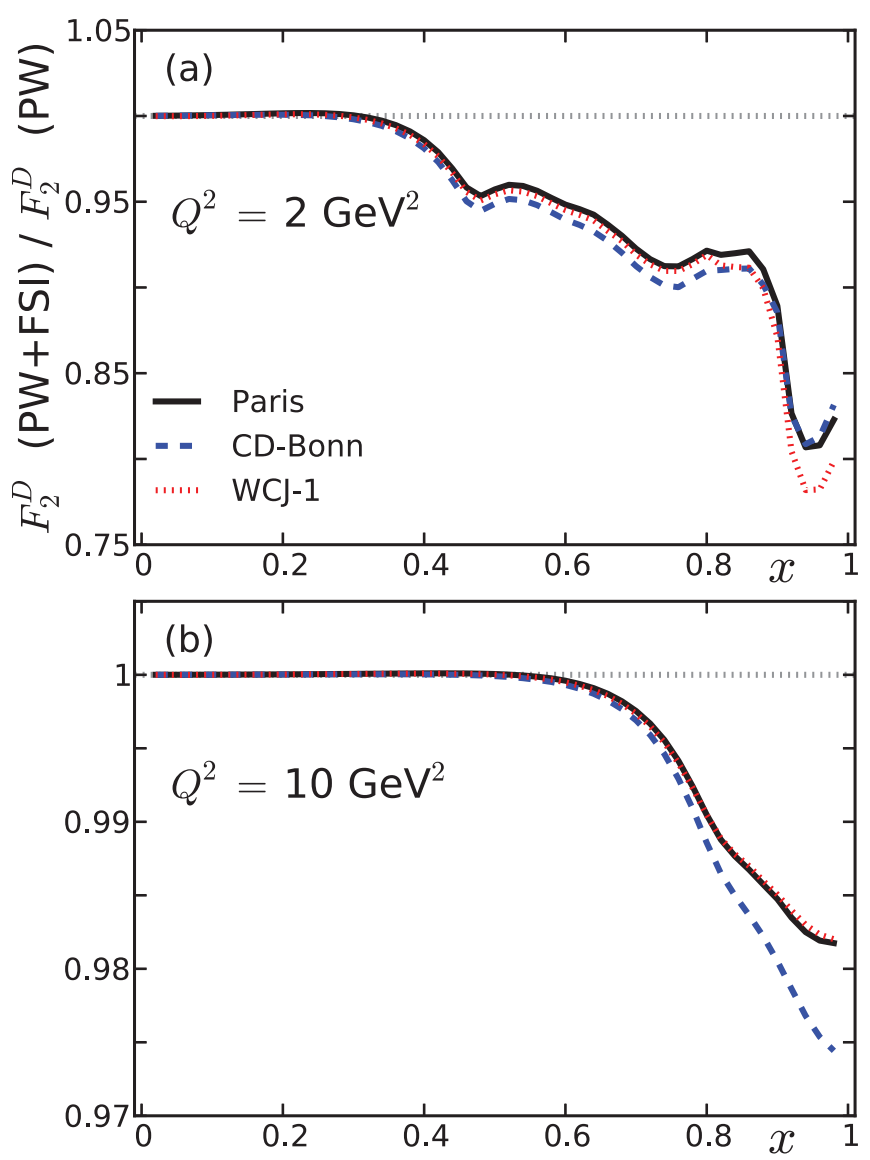

FIG. 8. (Color online) Ratio of the deuteron $F_{2}^{D}$ structure function with and without FSIs at $Q^{2}=2 \mathrm{GeV}^{2}$ (a) and $Q^{2}=20 \mathrm{GeV}^{2}$ (b). The results using the Paris deuteron wave function [57] (black solid curves) are compared with those employing the CD-Bonn [58] (blue dashed curves) and WCJ-1 [59] (red dotted curves) wave functions.

nucleon $F_{2}^{N}$ structure functions at $Q^{2}=2$ and $20 \mathrm{GeV}^{2}$ for the pw calculation and with FSIs using the on-shell and off-shell amplitudes. At the lower $Q^{2}$ value the resonance structures are clearly visible at large $x(x \gtrsim 0.4)$, with the ratios including FSIs slightly lower in magnitude compared with the pw results. With increasing $Q^{2}$ the resonance peaks migrate to higher $x$, revealing a smooth ratio with a trough at moderate $x$ values $(x \sim 0.6)$ and a large enhancement due to Fermi motion at $x \rightarrow 1$, typical of the "nuclear EMC effect" $[7,13,17,18,44]$. By $Q^{2}=20 \mathrm{GeV}^{2}$ the impact of the FSIs on the structure function ratio in the DIS region is negligible.

The sensitivity of these results to the specific model of the deuteron wave function used in the calculation can be seen in Fig. 8, where the ratios of the $F_{2}^{D}$ structure functions with and without FSIs are shown for the Paris [57], CD-Bonn [58], and WCJ-1 [59] $N N$ potential models. For comparison, we consider here the rescattering model involving only FSI contributions from the resonance region. This choice of wave functions spans the maximal range of effects possible using modern, high-precision $N N$ potentials [7]. Except at very high $x$ values $(x \gtrsim 0.8)$, where the $F_{2}^{D}$ is most sensitive to the short-range structure of the deuteron, the effects of the different 
deuteron wave functions is very small, in both the resonance and DIS kinematics. This result gives us some confidence that the findings of the FSI calculation are relatively robust and not strongly dependent on the details of the deuteron structure.

\section{CONCLUSION}

In this work we have presented a first quantitative analysis of the effects of FSIs in inclusive DIS from deuterium. Using the optical theorem and the properties of high-energy diffractive rescattering, a general result was derived within the GEA for the FSI contribution to the inclusive DIS deuteron cross section. To obtain numerical estimates of the FSI corrections we introduced a factorized model expressed through the bound-nucleon inelastic structure functions and the sum of hadronic rescattering amplitudes. The contributions to the rescattering amplitudes were modeled in terms of a set of three resonances with masses $W<2 \mathrm{GeV}$ and a continuum spectrum for the DIS region at $W \geqslant 2 \mathrm{GeV}$. The particular structure of these intermediate states was motivated by recent analyses [32] of spectator proton production data in SIDIS from the deuteron [34].

The formalism developed here includes on-shell and offshell contributions to the rescattering amplitude, the latter of which introduces some degree of model dependence. Importantly, however, we find that within this framework the FSI corrections vanish in the limit of large $Q^{2}$ because of phase-space constraints, independent of the details of the intermediate hadronic states. Numerically, we find sizeable FSI contributions to the inclusive $F_{2}^{D}$ structure function at $Q^{2} \lesssim 10 \mathrm{GeV}^{2}$ and $x \gtrsim 0.4$. Generally, the on-shell rescattering amplitude lowers the value of the structure function, with the effects most prominent at high $x$ and low $Q^{2}$, with a decrease of up to $20 \%$ found at $Q^{2}=2 \mathrm{GeV}^{2}$ as $x \rightarrow 1$.
The off-shell contributions to the rescattering generally enter with the opposite sign relative to the on-shell contributions, and in the $x \gtrsim 0.8$ region where their effects are most important they increase the magnitude of the deuteron structure function. Estimating the parameters that determine the off-shell contribution is difficult, however, and in our calculations we use the difference between the on-shell and off-shell results as an estimate on the uncertainty of the FSI corrections. In contrast, the dependence of the results on the deuteron wave function is found to be significantly smaller than the typical size of the FSI effects.

Our overall conclusion is that at $x \gtrsim 0.6$ and $Q^{2} \lesssim 10 \mathrm{GeV}^{2}$ the FSI effects can contribute to the deuteron $F_{2} \widetilde{D}$ structure function at the level of $2 \%-5 \%$ and should be considered in extractions of the neutron structure function from inclusive deuteron data at low $Q^{2}$. At larger $Q^{2}$ values $\left(Q^{2} \gtrsim 10 \mathrm{GeV}^{2}\right)$ in the deep-inelastic region the FSI effects are found to be negligible. Future data on tagged structure functions in SIDIS from the deuteron over a broader range of $Q^{2}$ and $W$ would be very helpful in further constraining the shape and magnitude of the FSI contributions to inclusive deuteron DIS. In this respect, the planned "BONuS" experiment [61] at the energyupgraded CEBAF accelerator at Jefferson Lab has the potential to provide new empirical guidance.

\section{ACKNOWLEDGMENTS}

W.C. is supported by the Research Foundation Flanders (FWO-Flanders). W.M. is supported by DOE Contract No. DEAC05-06OR23177, under which Jefferson Science Associates, LLC, operates Jefferson Lab. M.S. is supported by US Department of Energy grant under Contract No. DE-FG0201ER41172. The computational resources (Stevin Supercomputer Infrastructure) and services used in this work were provided by Ghent University, the Hercules Foundation and the Flemish Government, department EWI.
[1] L. W. Whitlow, E. M. Riordan, S. Dasu, S. Rock, and A. Bodek, Phys. Lett. B 282, 475 (1992).

[2] A. C. Benvenuti et al., Phys. Lett. B 237, 592 (1990).

[3] M. Arneodo et al., Nucl. Phys. B 487, 3 (1997).

[4] M. Arneodo et al., Nucl. Phys. B 483, 3 (1997).

[5] V. Tvaskis et al., Phys. Rev. C 81, 055207 (2010).

[6] A. Accardi, M. E. Christy, C. E. Keppel, W. Melnitchouk, P. Monaghan, J. G. Morfin, and J. F. Owens, Phys. Rev. D 81, 034016 (2010).

[7] A. Accardi, W. Melnitchouk, J. F. Owens, M. E. Christy, C. E. Keppel, L. Zhu, and J. G. Morfin, Phys. Rev. D 84, 014008 (2011).

[8] J. F. Owens, A. Accardi, and W. Melnitchouk, Phys. Rev. D 87, 094012 (2013).

[9] S. P. Malace, Y. Kahn, W. Melnitchouk, and C. E. Keppel, Phys. Rev. Lett. 104, 102001 (2010).

[10] A. D. Martin et al., Eur. Phys. J. C 73, 2318 (2013).

[11] R. D. Ball et al., Phys. Lett. B 723, 330 (2013).

[12] L. Frankfurt and M. Strikman, Phys. Lett. B 76, 333 (1978).

[13] S. A. Kulagin, G. Piller, and W. Weise, Phys. Rev. C 50, 1154 (1994).
[14] W. Melnitchouk and A. W. Thomas, Phys. Lett. B 377, 11 (1996).

[15] I. R. Afnan et al., Phys. Lett. B 493, 36 (2000).

[16] I. R. Afnan et al., Phys. Rev. C 68, 035201 (2003).

[17] M. M. Sargsian, S. Simula, and M. I. Strikman, Phys. Rev. C 66, 024001 (2002).

[18] S. A. Kulagin and R. Petti, Nucl. Phys. A 765, 126 (2006).

[19] Y. Kahn, W. Melnitchouk, and S. A. Kulagin, Phys. Rev. C 79, 035205 (2009).

[20] J. Arrington, J. G. Rubin, and W. Melnitchouk, Phys. Rev. Lett. 108, 252001 (2012).

[21] L. Frankfurt and M. Strikman, Nucl. Phys. B 316, 340 (1989).

[22] N. N. Nikolaev and B. G. Zakharov, Z. Phys. C 49, 607 (1991).

[23] V. R. Zoller, Z. Phys. C 54, 425 (1992).

[24] B. Badelek and J. Kwiecinski, Nucl. Phys. B 370, 278 (1992).

[25] W. Melnitchouk and A. W. Thomas, Phys. Rev. D 47, 3783 (1993).

[26] G. Piller and W. Weise, Phys. Rep. 330, 1 (2000).

[27] W. Melnitchouk, R. Ent, and C. E. Keppel, Phys. Rep. 406, 127 (2005) 
[28] J. C. Collins, Cambridge Monographs on Particle Physics, Nuclear Physics, and Cosmology (Cambridge University Press, New York, 2011).

[29] C. Ciofi degli Atti, L. P. Kaptari, and B. Z. Kopeliovich, Eur. Phys. J. A 19, 145 (2004).

[30] C. Ciofi degli Atti, L. P. Kaptari, and D. Treleani, Phys. Rev. C 63, 044601 (2001).

[31] V. Palli, C. Ciofi degli Atti, L. P. Kaptari, C. B. Mezzetti, and M. Alvioli, Phys. Rev. C 80, 054610 (2009).

[32] W. Cosyn and M. Sargsian, Phys. Rev. C 84, 014601 (2011).

[33] W. Cosyn and M. Sargsian, AIP Conf. Proc. 1369, 121 (2011).

[34] A. V. Klimenko et al., Phys. Rev. C 73, 035212 (2006).

[35] N. Baillie et al., Phys. Rev. Lett. 108, 199902 (2012).

[36] L. L. Frankfurt, M. M. Sargsian, and M. I. Strikman, Phys. Rev. C 56, 1124 (1997).

[37] M. Sargsian, Int. J. Mod. Phys. 10, 405 (2001).

[38] V. A. Abramovsky, V. N. Gribov, and O. V. Kancheli, Yad. Fiz. 18, 595 (1973).

[39] L. Frankfurt, M. Sargsian, and M. Strikman, Int. J. Mod. Phys. A 23, 2991 (2008).

[40] M. M. Sargsian, Phys. Rev. C 82, 014612 (2010).

[41] F. Gross, J. W. Van Orden, and K. Holinde, Phys. Rev. C 45, 2094 (1992).

[42] V. N. Gribov, Sov. Phys. JETP 30, 709 (1970).

[43] L. Bertocchi, Nuovo Cimento A 11, 45 (1972).

[44] W. Melnitchouk, A. W. Schreiber, and A. W. Thomas, Phys. Rev. D 49, 1183 (1994).
[45] V. R. Pandharipande and S. C. Pieper, Phys. Rev. C 45, 791 (1992).

[46] J.-M. Laget, Phys. Lett. B 609, 49 (2005).

[47] J. Ryckebusch, D. Debruyne, P. Lava, S. Janssen, B. Van Overmeire, and T. Van Cauteren, Nucl. Phys. A 728, 226 (2003).

[48] J. J. Aubert et al., Phys. Lett. B 123, 275 (1983).

[49] L. Frankfurt and M. Strikman, Nucl. Phys. B 250, 143 (1985).

[50] L. Frankfurt and M. Strikman, Phys. Rep. 160, 235 (1988).

[51] F. Gross and S. Liuti, Phys. Rev. C 45, 1374 (1992).

[52] M. R. Frank, B. K. Jennings, and G. A. Miller, Phys. Rev. C 54, 920 (1996).

[53] S. Boffi, C. Giusti, and F. Pacati, Phys. Rep. 226, 1 (1993).

[54] S. I. Alekhin, Phys. Rev. D 68, 014002 (2003).

[55] A. Bodek et al., Phys. Rev. D 20, 1471 (1979).

[56] M. E. Christy and P. E. Bosted, Phys. Rev. C 81, 055213 (2010).

[57] M. Lacombe, B. Loiseau, J. M. Richard, R. Vinh Mau, J. Conte, P. Pires, and R. de Tourreil, Phys. Rev. C 21, 861 (1980).

[58] R. Machleidt, Phys. Rev. C 63, 024001 (2001).

[59] F. Gross and A. Stadler, Phys. Rev. C 78, 014005 (2008).

[60] L. L. Frankfurt, M. I. Strikman, D. B. Day, and M. Sargsian, Phys. Rev. C 48, 2451 (1993).

[61] S. Bueltmann, M. E. Christy, H. Fenker, K. Griffioen, C. E. Keppel, S. Kuhn, and W. Melnitchouk, Jefferson Lab Experiment E12-10-102 [BONUS12], 2006. 